\title{
福辛普利钠预处理对缺血后适应大鼠心肌组织Toll样 受体表达及炎症因子的影响
}

\author{
张大武, 张蕾, 刘剑刚", 王承龙, 史大卓 \\ 中国中医科学院西苑医院心血管病中心, 北京 100091 \\ * 联系人, E-mail: liujiangang2002@sina.com
}

2009-12-11收稿, 2010-06-13接受

国家自然科学基金资助项目(30772868)

摘要大鼠预先灌胃福辛普利钠, 观察其和缺血后适应 (ischemic postconditioning, IPoC) 对大 鼠心肌缺血再灌注(ischemic reperfusion, I/R)损伤后心肌组织Toll样受体(toll-like receptor, TLR) 表达及心肌组织炎症浸润的影响. 将60只SD (Spragu-Dawley)大鼠随机分为假手术组、 $\mathrm{I} / \mathrm{R}$ 组、 $\mathrm{IPoC}$ 组以及福辛普利钠 $+\mathrm{IPoC}$ 组, 每组 15 只。假手术组大鼠心脏前降支下置线不结扎; $I / R$ 组予 以心脏前降支结扎 $30 \mathrm{~min}$, 再灌注 $1 \mathrm{~h} ; \mathrm{IPoC}$ 组给予心脏前降支结扎 $30 \mathrm{~min}$, 后予以 3 次 $10 \mathrm{~s}$ 的再 灌注/缺血循环, 再持续灌注 $1 \mathrm{~h}$; 福辛普利钠 $+\mathrm{IPoC}$ 组则预先给予福辛普利钠片 $0.9 \mathrm{mg} / \mathrm{kg}$, 连 续灌胃 $14 \mathrm{~d}$, 于末次灌胃 $2 \mathrm{~h}$ 后, 施以 $\mathrm{IPOC}$ 组的干预过程. 所有实验大鼠腹主动脉取血并分离出 心脏组织. 比色法测定血清肌钻蛋白 $\mathrm{T}$ (cardiac troponin T, cTNT) 的含量, NBT染色测定大鼠左 室心肌梗死面积, 酶联免疫吸附测定法测定心肌组织单核细胞趋化蛋白-1 (monocyte chemotactic protein-1, MCP-1) 和肿瘤坏死因子- $\alpha$ (tumor necrosis factor- $\alpha$, TNF- $\alpha$ ) 水平. 免疫组 织化学方法测定心肌组织TLR2及TLR4的表达, HE染色观察心肌组织炎性细胞的浸润. 结果表 明, 与 $\mathrm{I} / \mathrm{R}$ 组比较, $\mathrm{IPoC}$ 组大鼠心肌梗死面积明显缩小 $(P<0.01)$, 大鼠血清 $\mathrm{CTNT}$ 水平显著降低 $(P<0.01)$, 心肌组织 MCP-1, TNF- $\alpha$ 含量和炎性细胞浸润数量明显下降 $(P<0.05, P<0.01)$, 心肌 组织TLR2及TLR4的表达被显著抑制 $(P<0.01)$; 与 IPoC组比较, 福辛普利钠预处理可进一步降 低IPoC大鼠心肌梗死面积 $(P<0.05)$, 心肌组织TNF- $\alpha$ 水平也显著降低 $(P<0.01)$, 对心肌TLR2及 TLR4的表达有进一步抑制 $(P<0.05)$. 血管紧张素转化酶抑制剂福辛普利钠预处理可加强IPoC 对 $\mathrm{I} / \mathrm{R}$ 大鼠心肌的保护作用, 其机理可能与抑制TLR介导的炎症信号通路和趋化因子反应有关.

随着现代生活节奏的加快, 急性心肌梗死(acute myocardial infarction, AMI)已是严重危害人类健康的 世界性问题，其发病通常是在冠状动脉粥样硬化病 变的基础上继发斑块破裂，导致血栓形成，阻塞冠状 动脉, 相应的心肌组织持久的急性缺血所致. 因此, 建立绿色通道早期开通患者闭塞的冠状动脉, 有效 恢复缺血心肌的血流灌注, 是限制和缩小心肌梗死 面积、改善患者预后的关键措施. 近年来的临床研究 发现, 在冠状动脉再灌注开始时对冠脉进行短暂、重
复的开通及再闭过程，随后恢复冠状动脉血流，即 “缺血后适应”(ischemic postconditioning, IPoC), 对缺 血再灌注心脏有显著保护作用 ${ }^{[1]}$.

缺血后适应已被实验和临床研究证实可减轻再 灌注损伤心肌的梗死面积, 改善心脏功能 ${ }^{[2,3]}$. 药物 预处理对心肌缺血再灌注(ischemic reperfusion I/R) 损伤心肌的保护也有较多报道, 其中血管紧张素转 化酶抑制剂(angiotensin converting enzyme inhibitor, $\mathrm{ACEI}$ )对保护I/R心肌的作用较为明显 ${ }^{[4]}$, 但 ACEI预 
处理是否具有增加 IPoC 对 $I / R$ 损伤心肌的保护作用, 目前尚无报道. 缺血后适应心脏保护作用的机制尚 未完全阐明，本实验观察 $\mathrm{ACEI}$ 制剂福辛普利钠预处 理结合 IPoC 对再灌注损伤心肌的保护作用, 并从 Toll 样受体(toll-like receptor, TLR)及促炎性细胞因 子水平研究其作用机理.

\section{1 材料和方法}

(i ) 实验动物. 健康SD (Spragu-Dawley)大鼠, 清洁级, 60只, 雌雄兼用, 体重180 200 g, 由北京维 通利华实验动物技术有限公司提供, 合格证号: $\operatorname{SCXK}$ (京)2007-0001. 适应性饲养3 d 后进行实验, 动 物自由摄水，室温控制 $23 \sim 25^{\circ} \mathrm{C}$, 湿度为 $50 \% \sim 70 \%$, 光照 $12 \mathrm{~h}$, 黑暗 $12 \mathrm{~h}$.

(ii) 药物和试剂. 福辛普利钠片(商品名: 蒙 诺), $10 \mathrm{mg} /$ 粒，批号: 0804087, 中美上海施贵宝制药 有限公司生产. 肌钙蛋白 T (cardiac troponin T, cTNT) 试剂盒由美国RapidBio Lab公司生产，北京莱博特利 生物医学科技公司提供, 批号: 08060502; 单核细胞 趋化蛋白-1 (monocyte chemotactic protein-1, MCP1)、肿瘤坏死因子- $\alpha$ (tumor necrosis factor- $\alpha$, TNF- $\alpha$ ) 酶联免疫吸附测定 (enzyme-linked immunosorbant assay, ELISA)试剂盒、氯化硝基四氮唑兰(nitro blue tetrazolium chioride, NBT), 由美国R\&D (R\&D systems)公司生产. 考马斯亮蓝蛋白测定试剂盒, 由南 京建成科技有限公司生产，批号: 090828; Toll样受体 2 (toll-like receptor 2, TLR2)抗体试剂盒, Toll样受体4 (toll-like receptor 4, TLR4)抗体试剂盒, 由北京博奥 森生物技术有限公司提供. 浓缩型DAB试剂盒和免 疫组化试剂盒，由北京中杉金桥生物技术有限公司 提供.

( iii) 实验仪器. 动物人工呼吸机, 型号: DW-2000, 上海嘉鹏科技有限公司; 心电图机, 型号: ECG-6511, 上海光电仪器有限公司生产; Ultra Turrax T18高速分散机, 德国IKA公司; 7020型全自动生 化仪, 日本日立公司; Multiskan MK3型酶标仪, 荷兰 雷勃生物医学有限公司; DpxView Pro型显微彩色图 像处理系统, 丹麦Delta Pix公司.

(iv) 动物缺血后适应模型的建立. 大鼠以 $20 \%$ 氨基甲酸乙酯(乌拉坦) $6 \mathrm{~mL} / \mathrm{kg}$ 腹腔麻醉, 置于解剖 台上仰卧位固定，记录 II 导联心电图．颈部、胸骨左
侧、腹部消毒, 并剪毛备皮. 剪开颈部皮肤, 暴露气 管并插管, 连接动物呼吸机(潮气量 $3 \mathrm{~mL} / 100 \mathrm{~g}$ 体重, 呼吸频率为 60 次 $/ \mathrm{min}$ ), 沿胸骨左缘 $3 \sim 4$ 胁间开胸, 暴 露心脏, 剪开心包膜, 在左心耳的下缘、肺动脉圆雉 的左缘之间穿刺进针, 围绕左冠状动脉前降支上 $1 / 3$ 处穿3-0缝合线, 缝合线两端穿过一直径为 $1.5 \mathrm{~mm}$ 的 硅胶软管, 拉线推管, 蚊式止血钳固定以阻断左冠状 动脉血流. 结扎后 II 导联心电图示ST段明显抬高或T 波高尖, 结扎线下左室前壁呈暗红色为结扎成功. 30 $\min$ 后松开蚊式止血钳放松缝合线, 恢复冠状动脉血 流再灌注60 min. 后适应操作在结扎 $30 \mathrm{~min}$ 结束后, 立即给予 3 次 $10 \mathrm{~s}$ 的再灌注/缺血循环(松开蚊式止血 钳放松缝合线为再灌注，拉线推管用止血钳夹紧硅 胶管为缺血).

( V ) 动物分组及用药. 大鼠随机分为 4 组, 每 组15只：(1) 假手术组, 开胸冠状动脉前降支下置线 不结扎；(2) I/R组, 冠状动脉前降支结扎 $30 \mathrm{~min}$, 再 灌注 $1 \mathrm{~h}$ ，建立 $\mathrm{I} / \mathrm{R}$ 损伤模型；(3) IPoC组，建立 $\mathrm{I} / \mathrm{R}$ 模型 中予以3次 $10 \mathrm{~s}$ 的再灌注/缺血循环; (4) 福辛普利钠 $+\mathrm{IPoC}$ 组, 福辛普利钠, $0.9 \mathrm{mg} \cdot \mathrm{kg}^{-1} \cdot \mathrm{d}^{-1}$, 用等量蒸 馏水稀释后灌胃 $14 \mathrm{~d}$, 于末次灌胃 $2 \mathrm{~h}$ 后建立 I/R 模型, 过程中给予 $\mathrm{IPoC}$ 干预. 各组(除福辛普利钠 $+\mathrm{IPoC}$ 组 外)均给予等量生理盐水灌胃 $14 \mathrm{~d}$.

(vi) 动物样本的取材、分离和左室心肌梗死面 积测量. 完全松开缝合线再灌注 $60 \mathrm{~min}$ 结束后, 腹 主动脉取血并分离血清, 取出大鼠心脏生理盐水冲 洗干净后, 每组按随机数字表检测心肌组织指标, 5 只做NBT染色测量大鼠左室心梗面积, 5只液氮快速 冷冻后, $-80^{\circ} \mathrm{C}$ 冰箱保存用于组织ELISA检测, 5 只 $10 \%$ 中性甲醛溶液中保存用于免疫组织化学检测. 实 验中假手术组因灌胃死亡 1 只, I/R组造模中死亡 4 只, 其余2组死亡各2只, 后均予相应补充.

将取下的大鼠心脏用生理盐水冲洗干净, 滤纸 吸除多余水分, 均匀切成 5 片, 放人 $\mathrm{NBT}$ 染色液中, 置于 $37^{\circ} \mathrm{C}$ 水箱中温浴 $5 \mathrm{~min}$, 温浴过程中观察心肌组 织颜色, 当非梗死区显示蓝色, 梗死区为红色时, 取 出放置在滤纸上, 用Canon IXUS 90IS数码相机微距 拍摄后, 使用DpxView Pro型显微彩色图像处理系统 计算出左室梗死面积 $(\%)$, 用心肌梗死面积/左室面积 $\times 100 \%$ 表示.

(vii) 血清学指标和心肌组织MCP- 1 和TNF- $\alpha$ 含 量的检测. 比色法测定大鼠血清cTnT水平. 每组 5 只心脏，取前降支结扎线下 $3 \mathrm{~mm}$ 至心尖部左室缺血 
心肌组织 $100 \mathrm{mg}$, 加人 $2 \mathrm{~mL}$ 低温生理盐水 $\left(0^{\circ} \mathrm{C}\right), \mathrm{T} 18$ 高速分散机匀浆后, 离心取上清液, 于 $-80^{\circ} \mathrm{C}$ 保存待 测. 所有标本均采用ELISA法检测, 操作过程严格按 说明书操作程序进行. 同时用考马斯亮蓝蛋白测定 法检测每个组织标本的蛋白含量, 得出数值后, 将每 毫升匀浆液中的细胞因子含量换算成每毫克蛋白中 的细胞因子含量.

(Viii) 心肌组织TLR定位和免疫组织化学检测. 心肌标本用 $10 \%$ 中性缓冲甲醛液固定 $18 \mathrm{~h}$. 脱水、透 明、浸蜡、石蜡包埋. 连续切片约 $5 \mu \mathrm{m}$ 厚, 捞片于多 聚赖氨酸防脱处理过的载玻片上. 根据 $\mathrm{SABC}$ 试剂盒 说明书操作, 大鼠心肌切片常规脱腊, 封闭内源性过 氧化物酶, $10 \%$ 正常山羊血清封闭. 分别滴加一抗 TLR2抗体 $(1: 200)$ 和TLR4抗体 $(1: 800), 37^{\circ} \mathrm{C}$ 孵育 $1 \mathrm{~h}$, 磷酸缓冲液 $(\mathrm{PBS})$ 冲洗; 滴加二抗生物素化山羊抗小 鼠 $\mathrm{IgG}$, 室温 $20 \mathrm{~min}, \mathrm{PBS}$ 溶液冲洗; 滴加 $\mathrm{SABC}$ 复合 物, 室温 $20 \mathrm{~min}, \mathrm{PBS}$ 溶液冲洗; DAB- $\mathrm{H}_{2} \mathrm{O}_{2}$ 显色, 苏 木素轻度复染; 乙醇脱水、透明、中性胶封片, 选取 同批染色切片进行光学显微镜下观察. 每张切片随 机选取 3 个不重叠的视野 $(\times 400)$, 每只共计 15 个视野, 以心肌炎症部位的胞浆染成棕褐色为阳性细胞标志, 经病理图像计算机分析系统半定量分析大鼠心肌组 织阳性细胞的表达, 以阳性细胞面积占所有细胞面 积百分比表示结果.

(ix) 统计学处理. 所有数据以均数土标准差 $(\bar{x} \pm s)$ 表示, 使用SPSS16.0软件进行统计学分析. 单 因素方差分析各组别之间的差异, 组间两两比较采 用LSD法, $P<0.05$ 表示差异有统计学意义.

\section{2 结果}

\section{1 福辛普利钠预处理结合IPoC对大鼠血清cTnT 和心肌梗死范围的影响}

与假手术组比较, 各组大鼠心肌损伤后 $\mathrm{cTnT}$ 有 显著升高 $(P<0.01)$, 与 $\mathrm{I} / \mathrm{R}$ 组比较, IPoC组 $\mathrm{cTnT}$ 有显著 降低 $(P<0.01)$. 定量组织学NBT染色法显示左室心肌 梗死范围, I/R 组左室梗死面积为 $35.28 \% \pm 3.85 \%, \mathrm{IPoC}$ 组减少到 $21.02 \% \pm 2.29 \%(P<0.01)$, 福辛普利钠 $+\mathrm{IPoC}$ 组和 $\mathrm{IPoC}$ 组相比, 可进一步缩小心肌梗死面积 $(17.17 \% \pm 3.12 \%, P<0.05)$ (表1).

\section{2 福辛普利钠预处理结合IPoC对大鼠心肌组织 Toll样受体 2, 4表达的影响}

假手术组可见少量棕褐色颗粒, I/R组心肌内棕 褐色颗粒明显增多. 与假手术组比较, $I / R$ 组心肌组织 TLR2，4的表达均有显著升高 $(P<0.01)$; 与 $\mathrm{I} / \mathrm{R}$ 组比较, IPoC组TLR2, 4 表达显著降低 $(P<0.01)$; 与 IPoC组比 较福辛普利钠 $+\mathrm{IPoC}$ 组可进一步抑制 TLR2, 4 表达 $(P>0.05)($ 表2, 图1和2).

\section{3 福辛普利钠预处理结合IPoC对大鼠心肌TNF- $\alpha$ 和MCP-1水平以及炎性细胞浸润的影响}

与假手术组比较, 缺血再灌注后炎性细胞因子 MCP-1和TNF- $\alpha$ 显著增高 $(P<0.01)$; 与 $\mathrm{I} / \mathrm{R}$ 组比较, $\mathrm{IPoC}$ 干预能显著降低TNF- $\alpha$ 和MCP-1 $(P<0.05, P<0.01)$; 在 此基础上, 福辛普利钠能进一步降低IPoC心肌TNF- $\alpha$ 水平 $(P<0.01)$, 对 MCP-1有降低趋势, 但统计学处理 尚不显著. 见表3.

$\mathrm{HE}$ 染色结果显示, 与假手术组比较, 缺血再灌 注后浸润的炎性细胞数显著增加 $(P<0.01)$; 与 $\mathrm{I} / \mathrm{R}$ 组 比较, IPoC 干预能显著降低浸润细胞数 $(P<0.01)$; 福 辛普利钠 $+\mathrm{IPoC}$ 有进一步减少炎性细胞浸润的趋势, 但统计学处理尚不显著(表 3, 图 3).

\section{3 讨论}

在药物干预 I/R 损伤中, 大量研究表明 ACEI 有

表 1 各组大鼠血清 cTnT 含量和心肌梗死面积 ${ }^{a)}$

\begin{tabular}{lcccc}
\hline \multicolumn{1}{c}{ 组别 } & $n$ & $\mathrm{cTNT} / \mathrm{ng} \cdot \mathrm{mL}^{-1}$ & $n \begin{array}{c}\text { 左室梗死区比例 } \\
(\%)\end{array}$ \\
\hline 假手术组 & 14 & $0.015 \pm 0.01$ & 5 & 0 \\
$\mathrm{I} / \mathrm{R}$ 组 & 11 & $4.41 \pm 0.93^{* *}$ & 5 & $35.28 \pm 3.85^{* *}$ \\
$\mathrm{IPoC}$ 组 & 13 & $2.53 \pm 0.51^{* * \Delta \Delta}$ & 5 & $21.02 \pm 2.29^{\triangle \Delta}$ \\
福辛普利钠 $+\mathrm{IPoC}$ 组 & 13 & $2.36 \pm 0.96^{* * \Delta}$ & 5 & $17.17 \pm 3.12^{\triangle \Delta \square}$ \\
\hline \multicolumn{2}{c}{ a)**, 与假手术组比较, $P<0.01 ; \Delta \triangle$, 与 $\mathrm{I} / \mathrm{R}$ 组比较, $P<0.01 ; \square}$, \\
与 IPoC 组比较, $P<0.05$
\end{tabular}

表 2 各组大鼠心肌 TLR2, 4 表达水平变化 ${ }^{\text {a) }}$

\begin{tabular}{|c|c|c|c|}
\hline 组别 & $n$ & TLR2(\%) & TLR4(\%) \\
\hline 假手术组 & 5 & $10.92 \pm 5.28$ & $12.37 \pm 6.64$ \\
\hline$I / R$ 组 & 5 & $45.12 \pm 7.53^{* *}$ & $49.33 \pm 9.52^{* *}$ \\
\hline IPoC 组 & 5 & $35.89 \pm 8.19^{\triangle} \triangle$ & $38.42 \pm 8.16^{\triangle \triangle}$ \\
\hline 福辛普利钠+IPoC 组 & 5 & $29.02 \pm 6.50^{\triangle \Delta \square}$ & $30.59 \pm 7.17^{\triangle \triangle \square}$ \\
\hline
\end{tabular}



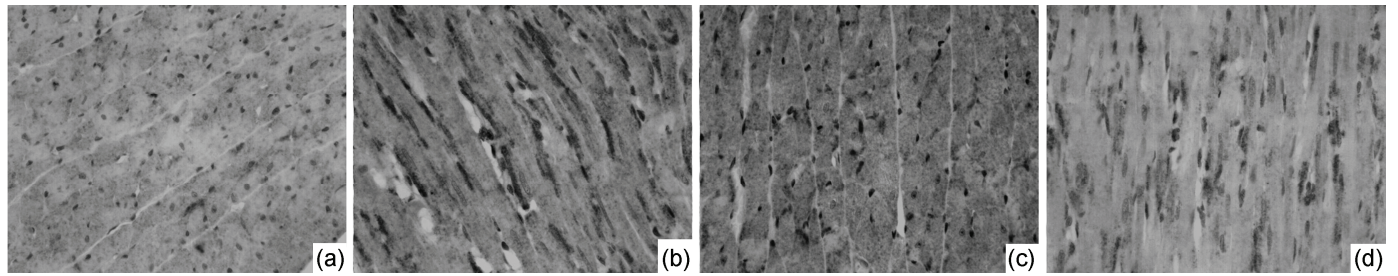

图 1 免疫组织化学法检测各组大鼠心肌 TLR2 表达(光学显微镜, $\times 400$ )

(a) 假手术组 $(n=5)$; (b) I/R 组 $(n=5)$; (c) IPoC 组 $(n=5)$; (d) 福辛普利钠 $+\mathrm{IPoC}$ 组 $(n=5)$
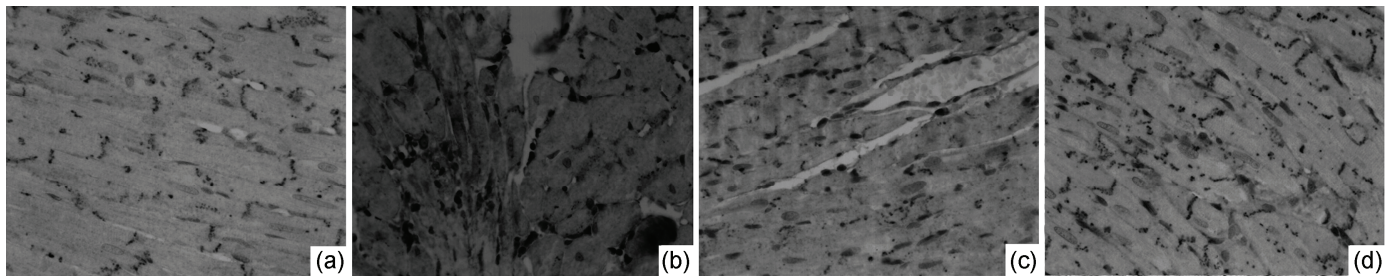

图 2 免疫组织化学法检测各组大鼠心肌 TLR4 表达(光学显微镜, $\times 400$ )

(a) 假手术组 $(n=5)$; (b) I/R 组 $(n=5)$; (c) IPoC 组 $(n=5)$; (d) 福辛普利钠 $+\mathrm{IPoC}$ 组 $(n=5)$

表 3 各组大鼠心肌 MCP-1, TNF- $\alpha$ 水平及炎性细胞浸润的影响 $(\bar{x} \pm s)^{\mathbf{a})}$

\begin{tabular}{|c|c|c|c|c|}
\hline 组别 & $n$ & $\mathrm{TNF}-\alpha / \mathrm{pg} \cdot \mathrm{mg}^{-1}$ & $\mathrm{MCP}-1 / \mathrm{pg} \cdot \mathrm{mg}^{-1}$ & 浸润细胞数(个) \\
\hline 假手术组 & 5 & $3.00 \pm 0.19$ & $12.60 \pm 3.68$ & $7.00 \pm 2.73$ \\
\hline$I / R$ 组 & 5 & $6.55 \pm 0.30^{* *}$ & $39.27 \pm 7.55^{* *}$ & $43.40 \pm 11.13^{* *}$ \\
\hline IPoC 组 & 5 & $4.71 \pm 0.27^{\triangle \triangle}$ & $29.97 \pm 2.29^{\triangle}$ & $24.80 \pm 4.44^{\triangle \triangle}$ \\
\hline 福辛普利钠+IPoC 组 & 5 & $4.21 \pm 0.32^{\triangle \triangle \square \square}$ & $25.50 \pm 4.50^{\triangle \triangle}$ & $22.60 \pm 4.88^{\triangle \Delta}$ \\
\hline
\end{tabular}

a) $* *$, 与假手术组比较, $P<0.01 ; \triangle, \triangle \triangle$ 与 $\mathrm{I} / \mathrm{R}$ 组比较, $P<0.05, P<0.01 ;$ 口, 与 $\mathrm{IPoC}$ 组比较, $P<0.01$
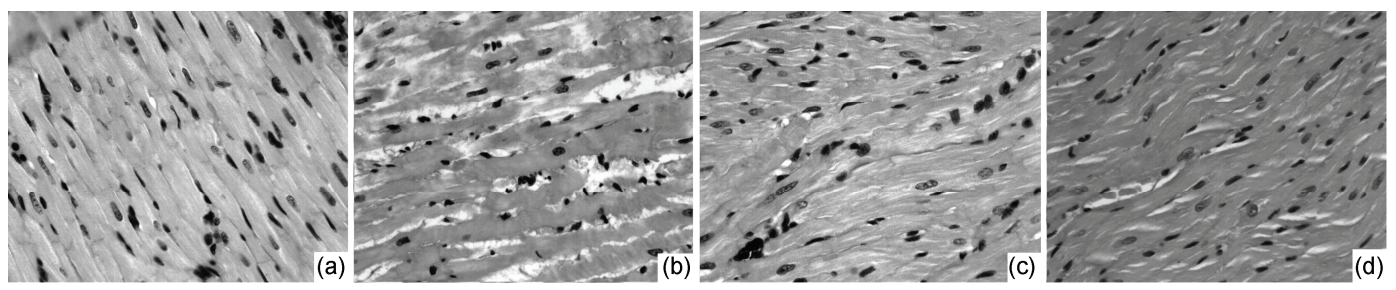

图 3 大鼠心肌 HE 染色浸润细胞数的变化(光学显微镜, $\times \mathbf{4 0 0}$ )

(a) 假手术组 $(n=5)$; (b) I/R 组 $(n=5)$; (c) IPoC 组 $(n=5)$; (d) 福辛普利钠 $+\mathrm{IPoC}$ 组 $(n=5)$. 黑色箭头所示为浸润细胞, 其中单核细胞为卵圆形, 细胞表面不规则, 细胞核长呈马蹄形, 或具浅的凹陷, 含有 1 2 个小的核仁; 淋巴细胞多为圆形或椭圆形, 核仁大而明显, 核内异染色质为主, 故核呈致密影

保护心肌免于 $I / R$ 损伤的作用 ${ }^{[4 ~ 6]}$. 给荷兰猪灌胃赖诺 普利 $10 \mathrm{~d}$ 后，再建立心肌 $\mathrm{I} / \mathrm{R}$ 损伤模型，结果显示 $\mathrm{I} / \mathrm{R}$ 荷兰猪心肌损伤明显减轻, 心脏功能显著改善 ${ }^{[5]}$. ACEI保护I/R心肌的机制可能与减少 $I / R$ 过程中大量 产生的血管紧张素 II 对心肌的损害有关 ${ }^{[6]}$. ACEI还 可使缓激肽生成增多, 分解减少, 促进前列腺素 $\mathrm{I}_{2}$ 和 一氧化氮生成, 用缓激肽受体拮抗剂后, ACEI抑制再 灌注心肌凋亡和减少心梗面积的作用明显减弱 ${ }^{[7]}$.
但是对于缺血再灌注损伤而言，其机制涉及活 性氧产生、多形核中性粒细胞聚集、钲超载和血管内 皮功能受损等诸多因素，以及随之诱导活化的信号 级联反应, 导致严重的心肌组织损伤和功能障碍. 其 表现出的是一种多因素、多途径的发病机制, 故单纯 的药物治疗对于缺血再灌注损伤, 未显示出有绝对 的优势. 既往研究也表明, 药物治疗干预缺血再灌注 损伤仍然停留于动物实验阶段，未能进人大规模临 
床实验. 近年来, $\mathrm{IPoC}$ 作为缺血再灌注损伤的辅助性 治疗手段被大量报道, 它有减少缺血再灌注损伤心肌 梗死面积和心肌损伤标志物释放的保护作用, 本研究 显示, IPoC能减少缺血再灌注大鼠心肌损伤标志物含 量和心肌梗死面积, 与既往研究相符, 那么药物治疗联 合缺血后适应是否能进一步减少心肌梗死面积, 改善 心脏功能, 目前尚未见报道. 我们的实验研究表明, 福 辛普利钠预先治疗, 可提高IPoC保护 $\mathrm{I} / \mathrm{R}$ 大鼠心肌作用, 较单纯IPoC可进一步缩小心肌梗死面积.

$T L R$ 是一组 I型跨膜受体, 通过与内、外源性配体 结合, 激发天然免疫和获得性免疫应答, 启动与免疫 和炎症相关的细胞因子IL- 6 , TNF- $\alpha$ 等基因的转录和 蛋白的表达 ${ }^{[8]}$. 有研究 ${ }^{[9,10]}$ 将小鼠TLR4基因敲除后建 立心肌 $\mathrm{I} / \mathrm{R}$ 损伤模型, 发现其与同种野生型小鼠相比 心肌梗死范围减少、炎症反应减轻、心肌梗死后的不 良重塑改善. 给予TLR4的抑制剂 eritoran干预后, I/R 后心肌梗死面积减少, 心肌组织NF-kappa B 和促炎 性细胞因子 MCP-1 和 TNF- $\alpha$ 水平降低 ${ }^{[11]}$. Sakata等 人 $^{[12]}$ 同样也发现TLR2缺陷小鼠遭受心肌 I/R损伤后 左室舒张末压较同种野生型小鼠下降, 促炎性细胞 因子TNF- $\alpha$ 和IL- $1 \beta$ 水平降低. 以上研究均表明抑制 TLR2，4的表达，可保护 I/R损伤心肌. 我们实验研究 发现, IPoC干预再灌注损伤大鼠心肌后TLR2, 4表达 降低, 心肌内MCP-1和TNF- $\alpha$ 含量也下降, 表明IPoC 抑制 TLR2，4的表达可能是其保护I/R心肌的一个重 要途径. 给予福辛普利钠预处理后, TLR2, 4的表达 与 IPoC组比较, 受到进一步抑制和降低. 心肌组织 TNF- $\alpha$ 的含量也显著下降, 表明福辛普利钠联合IPoC
可能与抑制TLR2, 4的表达和促炎性细胞因子的水平 有密切关系.

另外, 本实验研究显示心肌缺血再灌注时 MCP-1表达显著增加, 缺血再灌注心肌的炎性细胞 浸润也显著增多. 有研究 ${ }^{[13]}$ 表明, 将MCP-1特异性受 体 CCR2 基因敲除后, 小鼠缺血再灌注心肌炎性细胞 浸润显著降低, 心肌梗死面积缩小. 同样, 转染家兔 MCP-1抑制剂7ND基因抑制MCP-1活性后，再建立心 肌 I/R损伤家兔模型, 结果显示心肌炎性细胞浸润减 少, 促炎性细胞因子TNF- $\alpha$ 水平降低, 家兔心脏功能 明显改善, 表明抑制 MCP-1活性是保护 I/R心肌的重 要环节之一 ${ }^{[14]}$. 本研究应用 $I P O C$ 干预 $I / R$ 心肌后, 心 肌组织MCP-1 水平显著降低, 炎性细胞的浸润也明 显减少, 表明IPoC保护I/R心肌的作用与抑制 MCP-1 表达和炎性细胞浸润有关, 福辛普利钠联合 IPoC能 进一步降低MCP-1 水平和炎性细胞浸润, 统计学处 理尚不显著, 可能和样本量较少有关, 今后有待进一 步深人研究.

介人治疗技术已成为 AMI 再灌注治疗的重要手 段, 而缺血后适应的干预手段方法简便, 可操作性强, 对心肌缺血 I/R 的保护作用如能得以证实并进而在临 床应用, 可使患者在再灌注治疗中获得最大收益. 福 辛普利钠预处理与 IPoC 的联合应用, 可能为临床上 改善 AMI 早期再灌注治疗后患者的长期预后提供一 个重要的干预手段, 尤其是对有 AMI 高危因素诸如 高血压、糖尿病等疾病的患者, 预防性应用 ACEI 类 药物对于改善 AMI 再灌注损伤的心脏功能具有重要 意义。

\section{参考文献}

1 Zhao Z Q, Corvera J S, Halkos M E, et al. Inhibition of myocardial injury by ischemic postconditioning during reperfusion: Comparison with ischemic preconditioning. Am J Physiol Heart Circ Physiol, 2003, 285: H579-H588

2 Thibault H, Piot C, Staat P, et al. Long-term benefit of postconditioning. Circulation, 2008, 117: 1037-1044

3 Laskey W K, Yoon S, Calzada N, et al. Concordant improvements in coronary flow reserve and ST-segment resolution during percutaneous coronary intervention for acute myocardial infarction: A benefit of postconditioning. Catheter Cardiovasc Interv, 2008, 72: 212220

4 Ozer M K, Sahna E, Birincioglu M, et al. Effects of captopril and losartan on myocardial ischemia-reperfusion induced arrhythmias and necrosis in rats. Pharmacol Res, 2002, 45: 257-263

5 Doğan R, Farsak B, Isbir S, et al. Protective effect of lisinopril against ischemia-reperfusion injury in isolated guinea pig hearts. Cardiovasc Surg (Torino), 2001, 42: 43-48

6 Li K, Chen X. Protective effects of captopril and enalapril on myocardial ischemia and reperfusion damage of rat. J Mol Cell Cardiol, 1987, 19: 909-915

7 Wang L X, Ideishi M, Yahiro E, et a1. Mechanism of the cardioprotective effect of inhibition of the renin-angiotensin system on ischemia/reperfusion-induced myocardial injury. Hypertens Res, 2001, 24: 179-187 
8 Boyd J H, Mathur S, Wang Y, et al. Toll-like receptor stimulation in cardiomyoctes decreases contractility and initiates an NF-kappaB dependent inflammatory response. Cardiovasc Res, 2006, 72: 384-393

9 Oyama J, Blais C Jr, Liu X, et al. Reduced myocardial ischemiareperfusion injury in toll-like receptor 4-deficient mice. Circulation, 2004, 109: $784-789$

10 Riad A, Jager S, Sobirey M, et al. Toll-like receptor-4 modulates survival by induction of left ventricular remodeling after myocardial infarction in mice. J Immunol, 2008, 180: 6954-6961

11 Shimamoto A, Chong A J, Yada M, et al. Inhibition of Toll-like receptor 4 with eritoran attenuates myocardial ischemia-reperfusion injury. Circulation, 2006, 114: I270-I274

12 Sakata Y, Dong J W, Vallejo J G, et al. Toll-like receptor 2 modulates left ventricular function following ischemia-reperfusion injury. Am J Physiol Heart Circ Physiol, 2007, 292: H503-H509

13 Hayasaki T, Kaikita K, Okuma T, et al. CC chemokine receptor-2 deficiency attenuates oxidative stress and infarct size caused by myocardial ischemia-reperfusion in mice. Circ J, 2006, 70: 342-351

14 Kajihara N, Morita S, Nishida T, et al. Transfection with a dominant-negative inhibitor of monocyte chemoattractant protein-1 gene improves cardiac function after 6 hours of cold preservation. Circulation, 2003, 108(Suppl 1): II213-II218

\title{
Effect of fosinopril sodium pretreatment on toll-like receptor and inflammatory factor in rat myocardium with ischemic postconditioning
}

\author{
ZHANG DaWu, ZHANG Lei, LIU JianGang, WANG ChengLong \& SHI DaZhuo \\ \& CHEN KeJi
}

Department of Cardiology, Xiyuan Hospital, China Academy of Chinese Medical Sciences, Beijing 100091, China

\begin{abstract}
To observe the effects of ischemic post-conditioning (IPoC) on Toll-like receptor expression and myocardial inflammatory infiltration in myocardial tissue of rats with ischemic reperfusion (I/R) injury and fosinopril sodium pre-treatment, a total of 60 Sprague-Dawley rats were randomly assigned to the following groups: sham (suture around left anterior descending coronary artery was not tied), ischemic reperfusion (30 min in situ transient occlusion of the left anterior descending artery, followed by $1 \mathrm{~h}$ reperfusion), IPoC (30-min occlusion of left anterior descending artery, followed by three cycles of 10-s reperfusion/10-s ischemia prior to 1-h reperfusion), and fosinopril sodium +IPoC (fosinopril sodium pre-treatment, $0.9 \mathrm{mg} / \mathrm{kg}$, for $14 \mathrm{~d}$, followed by 2 -h I/R after last gavage, and IPoC treatment during I/R). Arterial blood and heart samples were extracted after 1-h reperfusion. Serum cTnT levels were measured using the colorimetric method, myocardial infarct size was measured using nitrotetrazolium blue/chloride staining, TNF- $\alpha$ and MCP-1 levels in myocardial tissue were measured by ELISA. TLR2 and TLR4 expression in myocardial tissue was analyzed using immunohistochemistry, and infiltrating cells were detected by hematoxylin-eosin staining. Compared with the I/R group, myocardial enzyme cTnT levels and infarct size significantly decreased in the IPoC group $(P<$ $0.01)$. In addition, MCP-1 and TNF- $\alpha$ levels, as well as the number of infiltrating cells in myocardial tissue, significantly decreased $(P<0.05, P<0.01$, respectively). TLR2 and 4 expressions also significantly decreased in the IPoC group $(P<0.05, P<0.01$, respectively). Compared with the IPoC group, the combination of fosinopril sodium and IPoC further reduced infarct size $(P<0.05)$, as well as TNF- $\alpha$ levels in myocardial tissue $(P<0.01)$. TLR2 and 4 expressions and infiltration of inflammatory cells were also significantly decreased $(P<0.05)$. Therefore, fosinopril sodium enhanced the protective effect of IPoC in a rat model of myocardial I/R injury. These mechanisms could be related to inhibition of the TLR signal pathway and chemotactic factor reactions.
\end{abstract}

fosinopril sodium, ischemic postconditioning, reperfusion injury, toll-like receptor, inflammatory factor

doi: $10.1360 / 972009-2610$ 